BULL. AUSTRAL. MATH. SOC.

VOL. 31 (1985), 433-437.

\title{
ON THE BASIS NUMBER OF SOME COMPLETE BIPARTITE GRAPHS
}

\author{
J. MCCALL
}

\begin{abstract}
This note completes the determination of the basis numbers of the complete bipartite graphs.
\end{abstract}

For a connected graph $G$, with $p$ vertices and $q$ edges, which is finite and does not contain loops or multiple edges, let $e_{1}, e_{2}, \ldots, e_{q}$ be an ordering of the edges. Then any subset $S$ of the edges corresponds to a q-dimensional vector $\left(a_{1}, a_{2}, \ldots, a_{q}\right)$ with $a_{i}=1$ if $e_{i} \in S$ and $a_{i}=0$ if $e_{i} k S$, for $i=1,2, \ldots, q$. These vectors form a vector space over the field $Z_{2}$. Those vectors which correspond to cycles of $G$ generate a subspace called the cycle space of $G$, denoted $\mathcal{C}(G)$. Strictly speaking the vectors generate $\mathcal{C}(G)$, but we usually think of the corresponding cycles as elements of the space.

A basis for $C(G)$ is called $k$-fold if each edge of $G$ occurs in at most $k$ cycles of the basis. The basis number of $G$, denoted $b(G)$, is defined as the minimum integer $k$ such that $G$ has a $k$-fold basis. Mac Lane [1] has shown that $G$ is planar if and only if $b(G) \leq 2$. Schmeichel [2] has investigated the basis number of some classes of nonplanar graphs and proved that $b\left(k_{n}\right)=3$ for $n \geq 5$, where $k_{n}$ denotes

Received 26 November 1984. The author is grateful to Dr C.H.C. Little for invaluable assistance with all aspects of this work.

Copyright Clearance Centre, Inc. Serial-fee code: 0004-9727/85 $\$$ A2.00 + 0.00 . 
the complete graph with $n$ vertices. He also established the basis number of most complete bipartite graphs $k_{m, n}$, for $m, n \geq 5, b\left(k_{m, n}\right)=4$, with the possible exceptions of $k_{5,5}, k_{5,6}, k_{5,7}, K_{5,8}, K_{6,6}, K_{6,7}, K_{6,8}$ and $K_{6,10}$. The remainder of this note constructs a 3 -fold basis for each of the eight graphs listed above, showing that the basis number is three in each case.

We note here that any 4 -cycle in $K_{m, n}$ is completely determined by its vertices, so will be denoted by listing them. We will represent the vertices in $X$ by numbers and those in $Y$ by letters, where $X$ and $Y$ are the two complementary, independent sets of vertices of $K_{m, n}$.

The method employed to find these 3 -fold bases is to modify the 4-fold bases given by Schmeichel using a sequence of transformations performed as follows. Consider a copy of $K_{2,3}$ in $K_{m, n}$. It has three cycles $C_{1}, C_{2}$ and $C_{3}$. At most two of these can belong to any basis of the cycle space of $K_{m, n}$. Suppose Schmeichel's basis contains two of them, say $C_{1}$ and $C_{2}$. Our transformation is to replace either of these by $C_{3}$, thereby yielding a new basis.

Now suppose $B$ is a basis of the cycle space of $K_{m, n}$ and $B$ contains $12 a b$ and $12 b c$. These cycles span a copy of $K_{2,3}$ and so $12 a b$ may be replaced by $12 a c$ according to our transformation. This replacement will be denoted by $12 a(b+c)$. The successive application of $12 a(b \rightarrow c)$ and $12 a(c \rightarrow d)$ will be denoted by $12 a(b \rightarrow c \rightarrow d)$. This notation can be extended in obvious ways.

If the letter labels are ordered, $a<b<c$ and so on, and we say $a+1=b, b+1=c$, and so on, then let $X^{\prime}$ (respectively $Y^{\prime}$ ) denote the set obtained from $X$ (respectively $Y$ ) by deleting the vertex with the highest valued label. The set of cycles of the form $x x+I \quad y \quad y+1$ with $x \in X^{\prime}$ and $y \in Y^{\prime}$ constitute Schmeichel's basis.

The sequences of transformations listed below yield 3-fold bases for the graphs in question, when applied to the bases given by Schmeichel. 


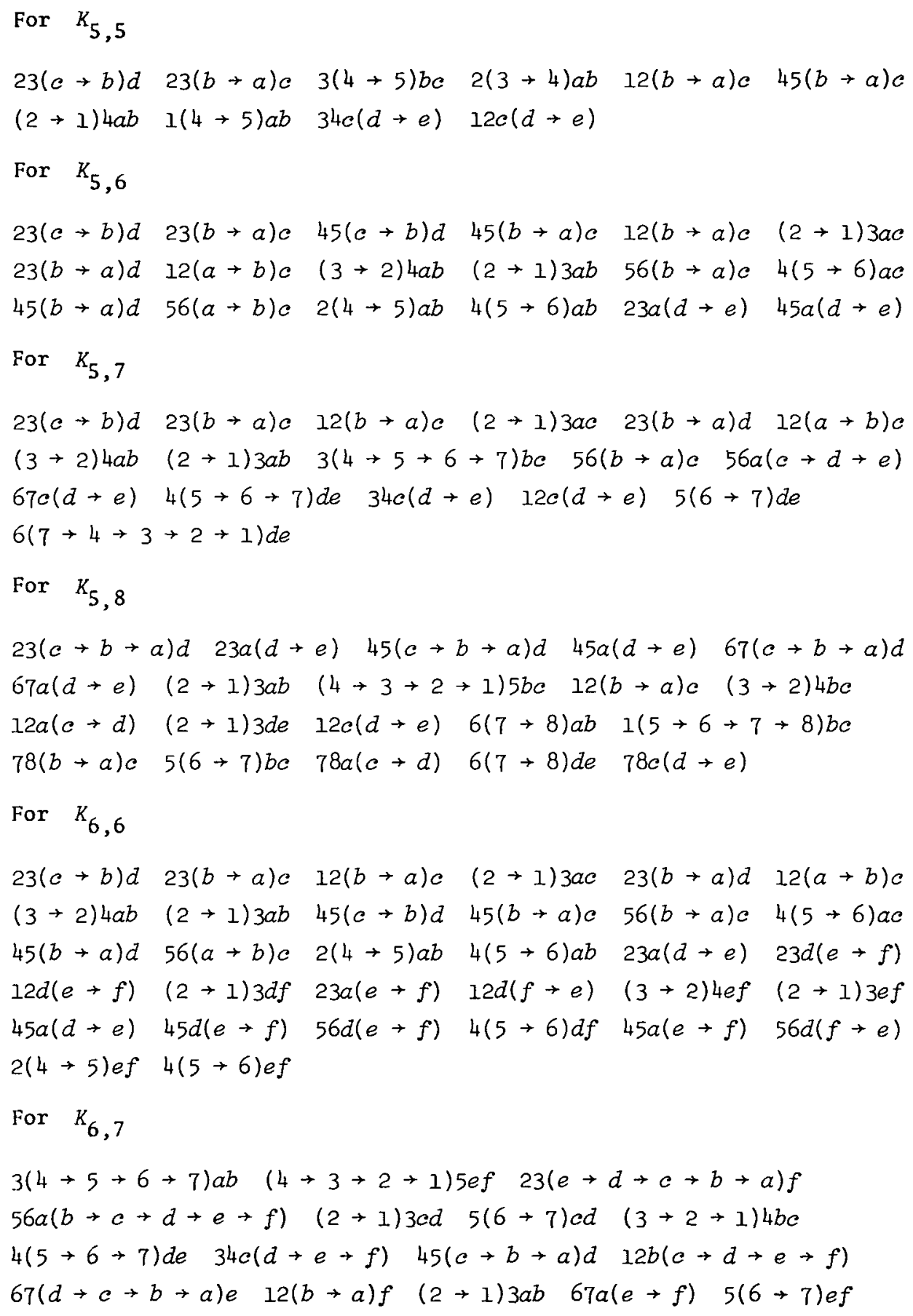




$$
\begin{aligned}
& \text { For } K_{6,8} \\
& (4 \rightarrow 3 \rightarrow 2 \rightarrow 1) 5 e d \quad 1(5 \rightarrow 6 \rightarrow 7 \rightarrow 8) c d \quad 23(c \rightarrow b+a) d \quad 23 a(d \rightarrow e+f) \\
& 67(c \rightarrow b \rightarrow a) d \quad 67 a(d \rightarrow e \rightarrow f) \quad 45(b \rightarrow a) c \quad 45 d(e \rightarrow f) \quad(3 \rightarrow 2+1) 4 a b \\
& (2 \rightarrow 1) 3 a b \quad 23 d(e+f \rightarrow a) \quad 12 a(b+c \rightarrow d \rightarrow e \rightarrow f) \quad(2 \rightarrow 1) 3 e f \\
& 5(6+7+8) a b \quad 6(7+8) a b \quad 67 d(e+f \rightarrow a) \quad 78 a(b+c+d \rightarrow e+f) \\
& 6(7 \rightarrow 8) e f \\
& \text { For } K_{6,10} \\
& (5+4+3+2) 6 c d \quad 2(6 \rightarrow 7 \rightarrow 8+9) c d \quad 23 c(d \rightarrow e) \quad 23(c \rightarrow b) e(2+1) 3 b c \\
& (2+1) 3 d e \quad(2+1) 3 a b \quad 12(b+a) c \quad(2 \rightarrow 1) 3 e f \quad 12 d(e+f) \quad(4 \rightarrow 3) 5 c d \\
& 34(b \rightarrow a) c \quad 34(c \rightarrow a) d \quad 34 d(e \rightarrow f) \quad 34 a(d \rightarrow f) \quad(3 \rightarrow 1 \rightarrow 2) 4 a b \\
& (3 \rightarrow 1 \rightarrow 2) 4 \text { ef } 2(4 \rightarrow 5) a b \quad 2(4 \rightarrow 5) \text { ef } 56 a(b \rightarrow c) \quad 56(e \rightarrow d) f(5+4) 6 b c \\
& (5 \rightarrow 4) 6 d e \quad 89(c \rightarrow b) d \quad 89 b(d \rightarrow e) \quad 8(9 \rightarrow 0) b c \quad 8(9+0) d e \quad 90(b+a) c \\
& 8(9 \rightarrow 0) a b \text { 90d }(e \rightarrow f) \quad 8(9 \rightarrow 0) \text { ef } 6(7 \rightarrow 8) c d \quad 78(b \rightarrow a) c \quad 78(c \rightarrow a) d \\
& 78 d(e \rightarrow f) \quad 78 a(d \rightarrow f) \quad 7(8 \rightarrow 0 \rightarrow 9) a b \quad 7(8 \rightarrow 0 \rightarrow 9) e f \quad(7 \rightarrow 6) 9 a b \\
& (7 \rightarrow 6) 9 e f \quad 4(6 \rightarrow 7) b c \quad 4(6 \rightarrow 7) d e
\end{aligned}
$$

These transformations yield the following 3-fold basis. The bases are listed in the order which results from Schmeichel's basis, that is when the cycle $C$ replaces cycle $C^{\prime}$ in the basis, the cycle $C$ is listed where $C^{\prime}$ would have been.

For $K_{5,5}$

$12 a b$ 12ac 12ce 12de $15 a b$ 23ac $23 b d$ 23de $34 a b$ 35bc 34ce $34 d e$ $45 a b \quad 45 a c 45 c d 45 d e$

For $K_{5,6}$

$12 a b$ 12bc 12cd 12de $13 a b$ 13ac 23ae 23de $25 a b$ 34bc 34cd 34de $46 a b$ 46ac $45 a e$ 45de 56ab 56bc 56cd 56de

For $K_{5,7}$

$12 a b$ 12bc 12ce 12de 13ab 13ac 23ad 23de $24 a b$ 37bd 34ce 34de $45 a b$ 45bc 45cd 47de 56ab 56ac 56cd 57de 67ab 67bc 67ce 16de 


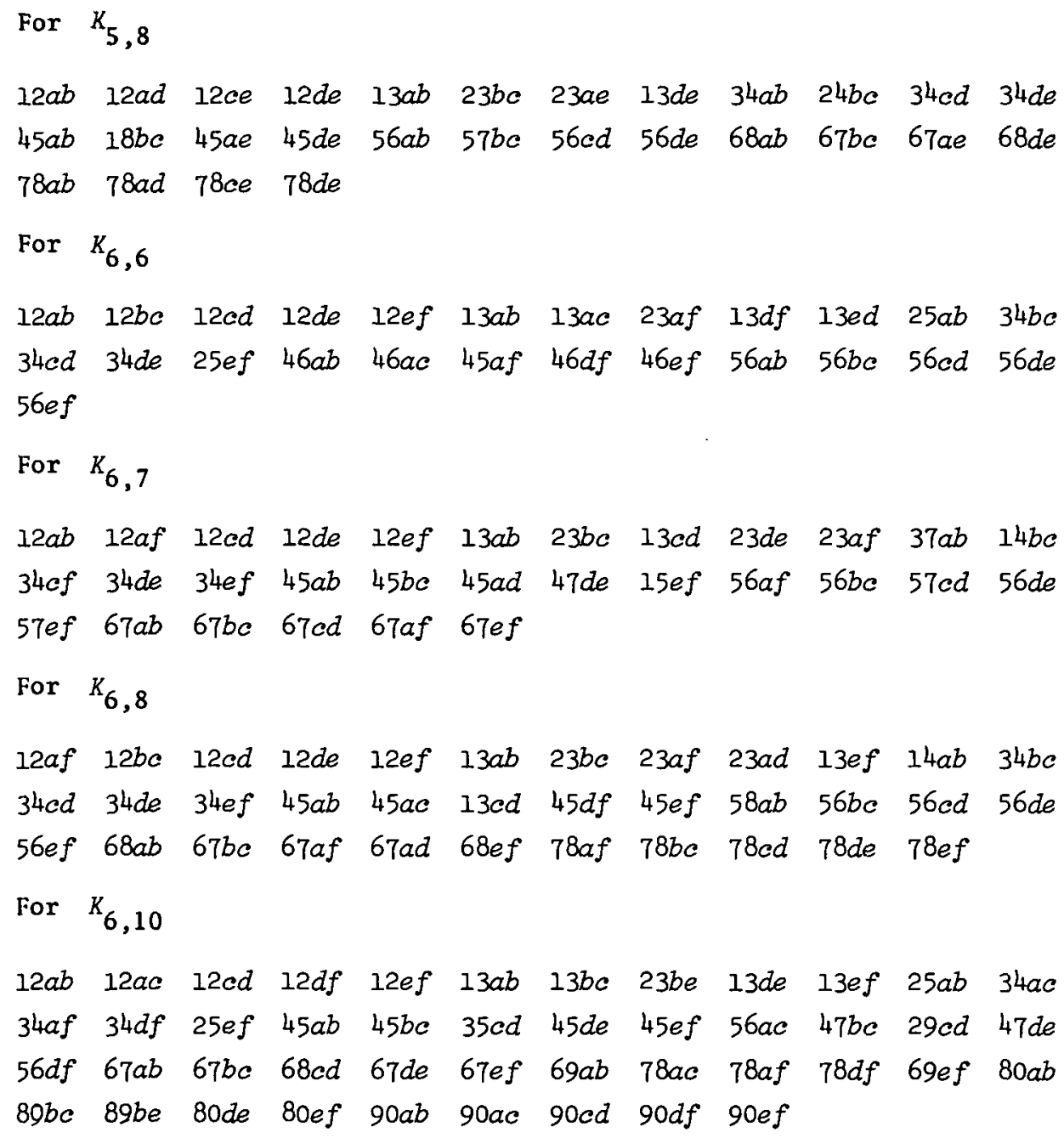

\section{References}

[1] S. Mac Lane, "A combinatorial condition for planar graphs", Fund. Math. 28 (1937), 22-32.

[2] E.F. Schmeichel, "The basis number of a graph", J. Combin. Theory Ser. B 30 (1981), 123-129.

100 Barrett Road, RD 4, New Plymouth, New Zealand. 